\title{
Factors influencing responsiveness to feedback: on the interplay between fear, confidence, and reasoning processes
}

\author{
Kevin W. Eva • Heather Armson • Eric Holmboe • Jocelyn Lockyer • \\ Elaine Loney $\cdot$ Karen Mann · Joan Sargeant
}

Received: 22 November 2010/Accepted: 11 March 2011/Published online: 6 April 2011

(C) The Author(s) 2011. This article is published with open access at Springerlink.com

\begin{abstract}
Self-appraisal has repeatedly been shown to be inadequate as a mechanism for performance improvement. This has placed greater emphasis on understanding the processes through which self-perception and external feedback interact to influence professional development. As feedback is inevitably interpreted through the lens of one's selfperceptions it is important to understand how learners interpret, accept, and use feedback (or not) and the factors that influence those interpretations. 134 participants from 8 health professional training/continuing competence programs were recruited to participate in focus groups. Analyses were designed to (a) elicit understandings of the processes used by learners and physicians to interpret, accept and use (or not) data to inform their perceptions of their clinical performance, and (b) further understand the factors (internal and external) believed to influence interpretation of feedback. Multiple influences appear to impact upon the interpretation and uptake of feedback. These include confidence, experience, and fear of not appearing knowledgeable. Importantly, however, each could have a paradoxical effect of both increasing and decreasing receptivity. Less prevalent but nonetheless important themes suggested mechanisms through which cognitive reasoning processes might impede growth from formative feedback. Many studies have examined the effectiveness of feedback through variable interventions focused on feedback delivery. This study suggests that it is equally important to consider feedback from the perspective of how it is received. The interplay observed between fear, confidence, and reasoning processes reinforces the notion that there is no simple recipe for the delivery of effective feedback.
\end{abstract}

\author{
K. W. Eva $(\bowtie)$ \\ Centre for Health Education Scholarship, University of British Columbia, \\ Vancouver, BC V5Z 4E3, Canada \\ e-mail: kevin.eva@ubc.ca \\ H. Armson · J. Lockyer \\ University of Calgary, Calgary, AB T2N 1N4, Canada \\ E. Holmboe \\ American Board of Internal Medicine, Philadelphia, PA 19106-3699, USA \\ E. Loney · K. Mann · J. Sargeant \\ Dalhousie University, Halifax, Nova Scotia B3H 4R2, Canada
}


These factors should be taken into account when trying to understand (a) why selfappraisal can be flawed, (b) why appropriate external feedback is vital (yet can be ineffective), and (c) why we may need to disentangle the goals of performance improvement from the goals of improving self-assessment.

Keywords Self-appraisal $\cdot$ Feedback $\cdot$ Confidence $\cdot$ Self-assessment $\cdot$ Performance improvement

\section{Introduction}

Much has been written in recent years about the inadequacy of self-assessment as an individually generated summary judgment of one's abilities. Dunning et al. (2004) published a monograph that reviewed the imperfect nature of self-assessment and the psychological basis for its inherent flaws; Eva and Regehr $(2005 ; 2008)$ conducted a broader exploration of the conceptual foundation of the term as it applies in health professional settings; and Davis et al. (2006) reported the results from a systematic review that indicated that the concerns identified in other domains and through studies with medical trainees do indeed apply to practicing physicians. The latter set of authors concluded that this literature "prompt[s] reflection on the use of self-rated assessment and its role in lifelong learning and value in self regulation and patient care."

The general conclusion from all of this effort is that accurate external feedback is crucial if one hopes to facilitate improvement (Sargeant et al. 2010). This conclusion is consistent with both the prevalent pleas from students for more feedback and with Boud's (1999) suggestion that the very concept of self-assessment should not be considered to be an individual activity, but instead, should encompass feedback from external sources. It is also consistent with Ericsson's (2004) model of deliberate practice which presents feedback and reflection as fundamental to the mechanism through which expertise is gained. For example, the insights of colleagues or supervisors regarding one's practice patterns might be expected to serve as information that can supplement personal opinions of one's own practice and there is ample evidence that such external feedback can improve performance (e.g., Boehler et al. 2006). Similarly, sitting an examination, be it written or clinical, provides feedback of a different, but equally powerful, type. Testing appears to have beneficial effects on memory/performance that extend beyond the potential benefits of repeated study (Larsen et al. 2008; Kromann et al. 2009). This latter form of external feedback is a specific example of a broader class of strategies for improving learning that Bjork has called "desirable difficulties" (1999). The notion here is that placing obstacles in front of learners, such as spreading training over multiple sessions (spaced training) rather than covering all of the to-be-learned material in a single session (massed training), creates challenges that implicitly provide feedback to learners that enables greater long-term gains in performance. Importantly, the benefit received is often counter to people's intuitions regarding which study conditions maximize learning (Boehler et al. 2006; Kornell and Son 2009).

Here the story becomes increasingly complicated in that feedback cannot generically be described as beneficial. The theory of desirable difficulties, and many empirical findings from that literature, suggest that increasing amounts of feedback has the potential to decrease learning as too much feedback can reduce the extent to which one needs to struggle with the material and, thus, can reduce long-term proficiency (Schmidt 1991). One factor that must be taken into account in trying to reconcile these varying views of the 
value of feedback is that feedback is not uniform in use or in concept. When, how, and by whom feedback is delivered matters and the effects are variable as a function of the specifics of the situation (Shute 2008; Kluger and van Dijk 2010). This is a salient point as we consider how feedback informs self-assessment and performance improvement.

It also leads to the broader realization that feedback of any form is never delivered or received in a vacuum. Unlike a technological system where feedback can be delivered as a command with immediate and uniform response, any feedback that is delivered will be interpreted through the filters with which the receiver views the world of practice, the feedback provider, and his/her own abilities. Additional filters include the receiver's motivations, fears, and expectations (Stewart 2008; Kennedy et al. 2009). As a result, one cannot take the 'self' out of the assessment if the goal is to have the information being delivered be deliberately incorporated (by the receiver) into practice. Previous examinations of the impact of multi-source feedback (MSF) support this statement as they have suggested that the extent to which feedback is deemed valuable is dependent, in part, on the degree to which the feedback can be reconciled with one's self-assessments (Sargeant et al. 2008). To take this a step further, it is important to question whether or not flawed self-appraisal must be corrected to enable performance improvement. For example, Kruger and Dunning's (1999) work demonstrates that performance gains often occur without altering individuals' self-perceptions, the two becoming aligned because performance increases to meet perceptions. This suggests that feedback is necessary, but doesn't necessarily need to be incorporated into one's self-concept to have an influence.

The purposes of this paper were to further explore the processes used by learners and physicians to interpret, accept and use (or not) data to inform their perceptions of their clinical performance, and to further understand the factors (internal and external) that appear to influence their interpretation of feedback. The aim is to better inform various educationally relevant activities including undergraduate and postgraduate training practices, remediation, continuing education, and knowledge translation.

\section{Methods}

The data analyzed to address these issues were collected as part of a larger qualitative study that took place across 5 countries using focus groups aimed at determining participants' perceptions of a variety of formal reflective activities (Sargeant et al. 2010). Using purposive sampling, we recruited learners at various levels of practice from 8 health professional training programs (within medicine and midwifery) known to use portfolios, personal learning plans, multi-source feedback, defined outcome competencies, and/or audit and feedback strategies. Programs included 3 undergraduate (midwifery and MD) programs, 2 postgraduate (medical residency) programs, and 3 practicing physician (family physicians and internists) programs. We invited volunteer participants by letter via each program's administrative office. Participating institutions' research ethics review boards approved the study.

We conducted 2 focus groups for 7 of the 8 programs and 3 focus groups for the 8 th program. They were about $1.5 \mathrm{~h}$ in length, recorded and transcribed. One researcher moderated all groups assisted by at least one team member or their research associate, a person familiar with the program and culture. Interviews were semi-structured and we used open-ended questions to explore participants' perceptions of self-assessment generally and the use of the specific program activities listed above with respect to their capacity to prompt pedagogically meaningful reflection that could inform one's self-assessment and 
direct learning. We used preliminary data analysis from each group to expand data collection and revise subsequent questions to address important concepts and emerging themes.

We conducted the analysis iteratively as a team. We developed an initial coding structure organized around the interview questions and emerging themes. We determined themes initially independently by open-coding transcripts and then comparing interpretations through group discussions conducted by teleconference and email. At least five team members analyzed each transcript and participated in this process, continually informing the coding structure and its revision. We resolved differences in interpretation through discussion and re-examining transcripts and coded data. We held a face-to-face meeting to confirm emerging themes and form subgroups to conduct detailed analyses of related categories of data. At a subsequent two-day meeting we discussed categories in depth, continued analysis through constant comparison, and constructed a preliminary conceptual diagram of informed self-assessment that was subsequently published (Sargeant et al. 2010). Analysis continued using constant comparison of incidents, categories and participant groups to elaborate the themes pertaining to the uptake and interpretation of data. High levels of team member engagement and interaction through all stages of analysis enhanced its rigour (Liamputtong and Ezzy 2005) as did the collaboration of team members from a variety of backgrounds. The current paper reports on a more detailed analysis of the data relevant to ways in which individuals reported using (or not using) external feedback, especially focusing on factors that influenced their interpretation of such information.

\section{Results}

A total of 134 participants took part in the 17 focus groups: 53 undergraduate learners, 32 postgraduate learners and 49 physicians. The ways in which participants interpreted external feedback, both formal and informal, fell into several categories. We discuss these as (1) the interplay between experience, confidence, and fear of not appearing knowledgeable; (2) influences upon accessing, interpreting and using feedback; and (3) mechanisms guiding (and potentially biasing) interpretation of external feedback.

The interplay between experience, confidence (real and projected), and fear of not appearing knowledgeable

Throughout discussions of the role of the self in judging one's performance and the readiness to accept feedback from other sources, it became clear that there remain fundamental differences of opinion across all groups with respect to how much learners and physicians should disclose to their patients, their colleagues, or themselves about their limitations. Some argued that lack of humility (i.e., the willingness to disclose one's limitations) is a fundamental problem with some individuals' practice.

You have to be able to say I made a mistake [or] I don't know. So it's kind of diametrically opposed to all of this training that you had that you are supposed to know and be confident and yet at the same time, you know you have to have this ability to look at yourself and say, I don't know. [Physician M2]

Others argued that one should not disclose this lack of knowledge to patients as desirable features of practice to be strived for include independence and the projection of confidence. 
I always tell people that there is nothing they can bring me that I can't figure out ... every symptom that they bring we will find a solution for ... That may not even be true, but I tell them that with confidence. [Physician M3]

I had a patient have a PSA and I had never heard of a fractionated PSA and he had read about it on the internet and wanted to know about it. I said I had no idea. Found out pretty quickly, but not quick enough as he was gone from my practice immediately. [Physician M3]

Such situations were especially challenging for learners. They described trying to assess their competence in these challenging situations vis-a-vis the risk to the patient and the ramifications of appearing incompetent to others if they called for help.

You don't want to show people you're not capable of doing this. So that part of calling the doctors [when uncertain], I tend not to. Maybe [calling the doctors is] a good way, but it also makes me feel like I'm too weak to do it myself, so I try to do figure it out by myself. [Medical student I1]

That said, physicians too were susceptible to fear of not appearing knowledgeable and suggested that the fear of appearing not to know is a motivational factor, one participant expressing "the fear of looking really stupid." [Physician L8].

With respect to enabling actual confidence, participants voiced opinions indicating that a large number of factors influenced their perceptions of their own performance, their motivations for wanting to perform well and their interpretation of externally generated information. A central and critical theme was that of experience. Experience was treated as a fundamental determinant of whether or not one is able to perform certain tasks. Both the absolute number of cases one has seen and the occurrence of specific experiences were thought to play a guiding role. However, little was mentioned in terms of the quality of either the experience or of the information gained from these experiences. That is, experience alone seemed to contribute to confidence and a sense of comfort.

If we had a particular number of patients whom I've [seen in] enough numbers, I'm confident that I can make most of the decisions without consulting my [senior] resident or attending. [Postgraduate O7]

For learners, experience was generally viewed positively, was desired, and generally led to increased confidence. Yet, confidence appeared tenuous and participants' comments also made it clear that loss of confidence in response to particular events is something that needs to be overcome.

I think you lose confidence in yourself if you do something well and all of a sudden ... you get a difficult one and then another difficult one, and then it's, you know what, I can't do that any more. [Postgraduate E5]

I can lose my confidence quite quickly, so I know that I could just put it off and avoid it and get other people to do it, but instead I try and bite the bullet and do it straight away, and then usually you're really concentrating the next time, you think, I've gotta bloody get this one, and then you do it and then it's fine. [Postgraduate E4]

"[T]he OSCE went really badly for me and I thought, oh, this is so bad that I don't wanna think about it anymore. Like, the next OSCE's miles away so I'll worry about it later. But I did a portfolio piece on it and I kind of split up all the things that went wrong and thought of ways that, to fix it for next time. And if I hadn't done portfolio 
I would've just left it and then probably [I'd] be in the same mess the next time round. [Medical student B6]

Indeed, confidence in one's abilities, both in terms of its fragility and participants' desire for it was a major theme when considering both trainees' and physicians' statements regarding the role of feedback. While participants clearly wanted feedback from others a number of statements implied that the desire could often be construed as wanting reassurance (i.e., as a source of confidence building rather than as a behaviour correction strategy).

I think I would rather have somebody else assess me than assess myself ... 'cause it just gives me a bit more confidence that what I'm doing is right. [Medical student C6]

It sounds weird to be alone in such a big group of people, but you do feel like you kind of need someone to go 'Yeah, that was great.' [Medical student B3]

That is not to say that participants valued confidence at all costs as many did speak of the risk of becoming over-confident and described how one's confidence needs to be informed by honest and humble self-appraisals of how they are doing:

"You know, if you think that you should be able to handle all things at all times, then you're going to get into trouble, and you have to recognize, and that's basically what self-assessment is, like, 'I'm not terribly good at this, I don't really get it all the time, and I need to know how to ask for help with that. [Physician A5]

In fact, participants' responses suggested a paradox in that one needed to achieve a particular level of comfort, experience, and confidence prior to being prepared to ask for or receive corrective feedback. That is, confidence and experience appeared to work in two ways: By enabling participants to ask for and accept feedback and, for some, guarding them against feedback that might be critical in nature.

If you feel bad and you feel uncomfortable, you're gonna not have the confidence to talk about mistakes, because if you already think that you're a rubbish doctor, and then you do something which probably ... is quite minor, but you know, you don't get any kind of constructive feedback because you never say to anybody, you know, because you're not comfortable saying it. [Postgraduate E6]

It might be a little paradoxical, the longer you are in practice the more prepared you are to admit you don't know. Because, I think at that point, you don't really care what people think, because hopefully you have developed a self esteem that is impervious to criticism. [Physician L8]

Influences on interpretation and uptake of external feedback

Some participants were cognizant that confidence alone could be an unreliable indicator of ability. A number of physicians spoke of the need to be shaken out of a prior belief and used emotionally-tied words like "shock" to express the feeling that first arises when discovering that there was a weakness in their performance. They further indicated a need to get over that feeling before the information could be utilized.

I don't think that we are any more than human, and I think that you do get into ruts.

... But I think there are people who just end up in ruts for a whole bunch of different 
reasons, and they know, they sorta stop thinking. It does require them to get a little bit of a jolt. [Physician J4]

So you get that initial shock, but then you think about it and look at are there potential reasons for this, or ... you think about a potential solution. So you go beyond just, 'what do [my colleagues] know.' [Physician J2]

If it's negative it always feels a bit painful. But it's true, when you look upon yourself and you know she's right, you can place it in a way. [Midwifery student G7]

The likelihood of such external information being utilized was very clearly thought to depend on the perceived credibility of the source, another dominant theme in the data. Emphasis was placed on the perceived accuracy of the feedback. Student participants discounted feedback provided by supervisors and others if they had not actually observed their performance. Family physicians likewise commented on critical consultants who did not understand the family physician's role and patients who lacked the medical knowledge or insight required to objectively judge care. Some noted that the lack of opportunity to readily engage in collegial interactions creates difficulty "when you are in ... solo practice" [Physician K7] and limits access to external feedback. Equally important was the need to believe that the feedback was delivered from a position of beneficence and nonmaleficence, that belief often developing as a result of strong relationships being established between the feedback deliverer and recipient.

And it doesn't mean that it is nice to hear it. It's not so nice; you don't feel so good after it. But it's a difference when if it's real, if what they're saying is just to help you, or if it's just to put you down. [Midwifery student F1]

If you have a supervisor who doesn't care or that doesn't give any feedback, then you aren't going to work on it. [Medical student H3]

I think it depends on the consultant who you're talking to. If it's someone who really knows you and who has taken interest in you before, then you'll be more willing to share and be honest, whereas if it's someone you've seen once or twice, then you might be a bit hesitant to actually say that. [Medical student C7]

Most people don't want to be exposed for their deficiencies unless they are comfortable with you. [Physician M3]

Mechanisms guiding (and potentially biasing) interpretation of external feedback

Throughout this discussion it appeared that respondents had any number of reasons to doubt the veracity of the external feedback received, thus reinforcing the notion that one must consider the recipient's interpretation of the feedback conveyed if one hopes to alter the focal behaviour. This doubt was outright in some instances:

I think it's quite difficult 'cause like with examination stuff literally everyone does it differently ... you can assess how good you are by kind of how confident you feel in what you're doing, but you'll never ... do it exactly the same as someone else who might, you know, might say, 'Oh, this is the right way.' [Medical student C3]

In other instances, the doubt indicated by participants' statements revealed potentially biased reasoning. That is, while the research design did not allow assessment of the accuracy of participants' statements, their choice of words were sometimes suggestive of 
ways in which reasoning about one's own performance could be led astray. For example, some expressed views that suggested a tendency to trust positive outcomes/feedback while discounting negative ones, thereby potentially increasing confidence by not accounting for the fact that positive outcomes can arise by chance just as readily as can negative outcomes. The relevant phrases have been underlined.

I was surprised that he gave me really positive feedback. And so that was useful to me because that was a real confidence booster when I wasn't expecting it at all. But that's about the only time I've found [feedback] useful. [Postgraduate E4]

I mean sometimes you'll do it and somebody will watch you and it didn't work and they're like, 'You did everything right, it's just one of those things.' But if it's

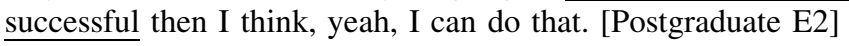

Adding to the potential for bias were statements reminiscent of the tendency humans have to attribute negative outcomes to situational (external) factors while attributing positive outcomes to one's own skill.

...then sometimes you're nervous, you're always nervous when [your supervisor] comes. Then you do something wrong and you get this strange reaction and you start doing everything wrong. Afterwards the patient is well: I think he did well, but then the mentor says that it really wasn't good enough. Then you feel hopeless because you did your best but everything went wrong because of the circumstances. [Midwifery student G2]

Some days I'll go home and say I was a really good doc today and feel good about what happened in the day. And then other days you know, if I have a headache, maybe I wasn't so good today. [Physician N7]

Finally, despite the discussion being focused on the use of external information to facilitate self-assessments, many participants made overarching statements about their confidence in their experience and "knowing enough." These statements suggested that they remained of the opinion that at some fundamental level they were able to judge for themselves whether or not they are fit to practice in specific situations.

I think there are a lot of things I don't know, but I think I know enough to make sure the patient doesn't crash. [Postgraduate Q7]

That to me is a very important part of my self assessment where I feel that I know enough to know when to worry. [Physician L8]

\section{Discussion}

This paper explored the interpretation of external feedback and its assimilation with selfperceptions. The dominant influence appeared to be confidence, which allows one to hear potentially threatening appraisals but also gets in the way because people think they have to project it. Additionally, that same confidence may lessen the extent to which external feedback that is inconsistent with one's self-appraisals is deemed credible. Experience (i.e., the number of times doing an activity) was directly linked to developing a sense of confidence. Importantly, however, confidence was fragile and participants valued feedback mechanisms that maintained confidence in a way that is reminiscent of the self-efficacy literature's demonstration of the value of positive feedback (Bandura 1997; Teunissen et al. 
2007). Through all this it was clear that, when conflict arises between external data and one's own self-appraisal, attempts to reconcile the dissonance are variable and complex. Most studies of the influence of feedback on performance have focused upon strategies through which feedback can be delivered (Shute 2008). That experience, confidence, fear, and biases inherent in cognitive reasoning processes can impact upon one's response to feedback (for better or worse) regardless of which strategy is used leads us to believe it is equally if not more important to consider how feedback is received and data are interpreted.

The social psychology literature provides useful insight to aid the interpretation of our findings. In this domain judgment and decision-making are typically considered through examination of what factors influence one's interpretation of data/an issue. Growing out of the classic work of Tversky and Kahneman (1974), researchers in this field have identified two broad classes of influences sometimes described as "hot cognition" and "cold cognition" (Gilovich 1991). Hot cognitions are related to emotion, arousal, and motivation, often involving factors that protect or strengthen one's perception of oneself. For example, self-serving bias is said to arise when people attribute failures of performance to situational factors and successes to their own ability. Cold cognition, in contrast, represents influences that arise more from analytic or unemotional ways in which attention, memory, and judgment operate. A well-known example of cold cognition is confirmation bias (i.e., the tendency to test hypotheses in a one-sided way, thus ensuring that the balance of evidence supports our preconceptions). The methodology used in this study does not allow a statement to be made regarding the accuracy of the participants' interpretations or the prevalence of actual "bias." However, the comments generated by participants suggest that both hot and cold cognition can influence one's perception of oneself and impact upon the way one interprets feedback from others just as readily as these influences have been found to impact upon people's perceptions of broader issues.

In terms of hot cognition, participants noted the emotional nature of seeking/receiving feedback. Wanting to develop and improve was balanced with fears of "looking stupid," giving a general sense that receivers/seekers of feedback were in a vulnerable position either with respect to losing patients, harming patients, or losing face with peers, supervisors, trainees, or oneself. The emotionally evocative nature of this activity and the selfprotective nature of participants' responses were further indirectly reflected in three ways: (1) participants' statements about requiring confidence prior to being prepared to receive feedback, (2) the notion that one is likely to be more open to feedback within the context of an established environment of trust in which the feedback provider presents from a position of beneficence and non-maleficence, and (3) the "shock" described as a common initial response that one must try deliberately to overcome to avoid shrinking away from tasks or situations in which performance was sub-optimal. This latter finding brings to mind the popular "stages of grief" models of coping with bad news (e.g., moving from anger through denial, bargaining, depression, and acceptance; Kübler-Ross 2005) and suggests that the educational community might do well to consider their relevance to the delivery of feedback in educational settings. That receiving feedback is not an emotionally neutral task requires us to consider the psychological immune system (cognitive mechanisms that protect the subject from negative emotions), as described by Gilbert and Wilson (2000), as one of the reasons whereby participants' stated desire for feedback may still, in some circumstances, translate into feedback not being sought (i.e., avoidance) or in its not being recognized as valuable when it is available (i.e., discounting).

We do not mean to imply that feedback is never heeded or used to guide adaptations in practice. Further, we readily acknowledge that it is perfectly appropriate to discount 
feedback in many circumstances. The argument laid out above, however, is meant to highlight that the adaptive nature of positive self-efficacy (Dweck 1999) makes it such that in many circumstances we are better off maintaining a positive outlook regarding our own abilities. As a result, we may be prone to things like self-serving bias, as seemed to be demonstrated by the few participants whose statements indicated a tendency to broadly attribute negative events to external factors like "headaches" and "other circumstances". That is not to say that participants were deliberately acting in a self-serving way or that these biases were inevitable and, indeed, we saw many instances of both positive and negative feedback being discounted by virtue of it being perceived as non-credible. Rather, it simply points out that the cognitive tendencies that have been readily identified in many other domains as biasing our reasoning in a personally adaptive manner must be considered in determining how feedback is used/interpreted. This further suggests that we, as educators, may be best served by separating the goal of performance improvement from the goal of accurate self-assessment as the latter may not be the causal path to the former.

We speculate that awareness, implicit or otherwise, of the hot cognition involved in interpreting feedback can contribute to the hesitation many teachers/peers feel regarding the need to deliver feedback. Removing the emotion from the situation, however, is unlikely to completely overcome the difficulties inherent in incorporating feedback. Indeed, participant interviews suggested that cold cognition (i.e., limitations of memory/ attention) is also involved. A dominant theme derived from participants' responses is that they tended to draw inferences about their ability from a count of how often they had experienced the specific case/task previously. That would seem appropriate, but noticeable by its absence was indication of the quality of those experiences in terms of what information was provided or what was actually learned. Previous work in both clinical and lay domains has suggested that, when left to our own devices, we judge ourselves to have achieved proficiency when the rate at which we are learning from additional experiences declines (i.e., rather than judging proficiency based on the actual level of performance achieved; Eva 2009). That experience determines confidence, therefore, becomes a doubleedged sword. Without experience some participants indicated discomfort with collecting feedback and concern about the negative impact it could have on their self-efficacy. As experience accumulates, however, one gains both confidence and a greater capacity to discount corrective feedback as a result of having a greater personal database of cases on which to draw as "evidence" that the skill has been achieved and that the source of the feedback (be it a person or a test or some other dataset) must be biased in some way or misinformed about the receiver's abilities. Again, this is not to suggest that such discounting of feedback is inevitable or that most participants did not have a true desire to improve and practice to the best of their abilities. Indeed, many participant comments make it clear that feedback is generally desired and that the problem is often that feedback is simply unavailable or truly of insufficient quality. Rather, the interviews and this theoretical perspective merely help us to understand the complex variety of ways in which feedback, when received, can be interpreted in ways that can lead to it falling on deaf ears despite the best intentions of all involved.

\section{Limitations}

On one hand it would seem counter-intuitive to attempt to study issues of relevance to the difficulty people have with judging their own knowledge/performance by asking them to discuss their knowledge/performance and the factors they perceive to influence them. The results of this study, however, reinforce the need to study these issues in this way. 
Feedback is never provided in a vacuum. As such, any effort to improve performance and overcome reliance on often flawed personal judgments must be considered in the context of what receivers believe provides important guidance regarding the credibility of that feedback. How feedback is perceived and discussed will determine how feedback is interpreted and adopted (Hattie and Timperley 2007). A greater limitation of the research reported here is that the researchers themselves are prone to biases in their interpretation of participant responses, the tendency to confirm one's preconceptions providing one obvious concern. We aimed to minimize this problem by having multiple team members read the transcripts and independently generate themes/ideas rather than relying on the perceptions of one individual. At the same time, we draw confidence from the fact that many of the themes identified were not anticipated to present themselves in this dataset. Furthermore, the findings align with well-established and empirically supported theories from a completely independent literature on social psychology of which most of our participants and most members of our research team were unaware. That a variety of methodologies have yielded similar conclusions across disparate domains can be seen as providing evidence of triangulation, thus strengthening claims that the results are meaningful and add to our understanding of how some basic psychological issues may impact upon professional selfregulation.

\section{Conclusion}

By way of summary, the interplay between fear (of looking stupid or of negative feedback), confidence (as derived from experience and influencing one's willingness to seek/ accept feedback), and reasoning processes (with their emotional and analytic aspects of hot and cold cognition, respectively) appears to create a complex mixture that reinforces the notion that there is no simple recipe for the delivery of feedback. While most teaching around feedback focuses on delivery, feedback providers must learn to take into account the self-perceptions of the individual being assessed. If one hopes to convey feedback that is perceived as credible an effort must be made to tailor feedback in a manner that will be interpretable and palatable through the lens of the recipient's perceptions. Feedback appears most likely to be perceived as worthy of attention and action when delivered from a clear position of beneficence that allows the learner to maintain their self-concept. To enable this it may be worth exploring how to efficaciously translate the notions of shared decision-making (Makoul and Clayman 2006) and relationship centred-care (Beach and Inui 2006), prevalent in the clinical world, into the educational process and the learnersupervisor relationship.

Open Access This article is distributed under the terms of the Creative Commons Attribution Noncommercial License which permits any noncommercial use, distribution, and reproduction in any medium, provided the original author(s) and source are credited.

\section{References}

Bandura, A. (1997). Self-efficacy: The exercise of control. San Francisco: Freeman.

Beach, M. C., \& Inui, T. (2006). Relationship-centered care: A constructive reframing. Journal of General Internal Medicine, 21, S3-S8.

Bjork, R. A. (1999). Assessing our own competence: Heuristics and illusions. In: Attention and performance XVII: Cognitive regulation of performance: Interaction of theory and application. Cambridge, MA: MIT Press. 
Boehler, M. L., Rogers, D. A., Schwind, C. J., Mayforth, R., Quin, J., Williams, R. G., et al. (2006). An investigation of medical student reactions to feedback: A randomized controlled trial. Medical Education, 40, 746-749.

Boud, D. (1999). Avoiding the traps: Seeking good practice in the use of self assessment and reflection in professional courses. Social Work in Education, 18, 121-132.

Davis, D. A., Mazmanian, P. E., Fordis, M., Van Harrison, R., Thorpe, K. E., \& Perrier, L. (2006). Accuracy of physician self-assessment compared with observed measures of competence: A systematic review. JAMA, 288, 1057-1060.

Dunning, D., Heath, C., \& Suls, J. M. (2004). Flawed self-assessment: Implications for health, education, and the workplace. Psychological Science in the Public Interest, 5, 69-106.

Dweck, C. S. (1999). Caution-Praise can be dangerous. American Educator, 23, 4-9.

Ericsson, K. A. (2004). Deliberate practice and the acquisition and maintenance of expert performance in medicine and related domains. Academic Medicine, 79, S70-S81.

Eva, K. W. (2009). Diagnostic error in medical education: Where wrongs can make rights. Advances in Health Sciences Education, 14, 71-81.

Eva, K. W., \& Regehr, G. (2005). Self-assessment in the Health Professions: A Reformulation and Research Agenda. Academic Medicine, 80(10 Suppl.), S46-S54.

Eva, K. W., \& Regehr, G. (2008). "I'll never play professional football" and other fallacies of selfassessment. Journal for Continuing Education in the Health Professions, 28, 14-19.

Gilbert, D. T., \& Wilson, T. D. (2000). Miswanting. In J. Forgas (Ed.), Thinking and feeling: The role of affect in social cognition (pp. 178-197). Cambridge: Cambridge University Press.

Gilovich, T. (1991). How people know what isn't so: The fallibility of reason in everyday life. New York: The Free Press.

Hattie, J., \& Timperley, H. (2007). The power of feedback. Rev Educ Res, 77(1), 81-112.

Kennedy, T. J., Regehr, G., Baker, G. R., \& Lingard, L. (2009). Preserving professional credibility: Grounded theory study of medical trainees' requests for clinical support. Medical Education, 43, $645-653$.

Kluger, A. N., \& van Dijk, D. (2010) Feedback, the various tasks of the physician, and the feed forward alternative. Medical Education 44, 1166-1174.

Kornell, N., \& Son, L. K. (2009). Learners' choices and beliefs about self-testing. Memory, 17, 493-501.

Kromann, C. B., Jensen, M. L., \& Ringsted, C. (2009). The effect of testing on skills learning. Medical Education, 43, 21-27.

Kruger, J., \& Dunning, D. (1999). Unskilled and unaware of it: How difficulties in recognizing one's own incompetence lead to inflated self-assessments. Journal of Personality and Social Psychology, 77, 1121-1134.

Kübler-Ross, E. (2005). On grief and grieving: Finding the meaning of grief through the five stages of loss. NY: Simon \& Schuster Ltd.

Larsen, D. P., Butler, A. C., \& Roediger, H. L., 3rd. (2008). Test-enhanced learning in medical education. Medical Education, 42, 959-966.

Liamputtong, P., \& Ezzy, D. (2005). Qualitative research methods (2nd ed.). Melbourne: Oxford University Press.

Makoul, G., \& Clayman, M. L. (2006). An intergrative model of shared decision making in medical encounters. Patient Educ Couns, 60(3), 301-312.

Sargeant, J., Armson, H., Chesluk, B., Dornan, T., Eva, K., Holmboe, E., Lockyer, J., Loney, E., Mann, K., van der Vleuten, C. (2010). The processes and dimensions of informed self-assessment. Academic Medicine Epub Apr 2, 2010.

Sargeant, J., Mann, K., van der Vleuten, C., \& Metsemakers, J. (2008). “Directed” self-assessment: Practice and feedback within a social context. Journal of Continuing Education in the Health Professions, 28, 47-54.

Schmidt, R. A. (1991). Frequent augmented feedback can degrade learning: Evidence and interpretations. In G. E. Stelmach \& J. Requin (Eds.), Tutorials in motor neuroscience. Kluwer: Dordrecht.

Shute, V. J. (2008). Focus on formative feedback. Review of Educational Research, 78, 153-189.

Stewart, J. (2008). To call or not to call: A judgement of risk by pre-registration house officers. Medical Education, 42, 938-944.

Teunissen, P. W., Scheele, F., Scherpbier, A. J., van der Vleuten, C. P., Boor, K., van Luijk, S. J., et al. (2007). How residents learn: Qualitative evidence for the pivotal role of clinical activities. Medical Education, 41, 763-770.

Tversky, A., \& Kahneman, D. (1974). Judgment under uncertainty: Heuristics and biases. Science, 185, 1124-1131. 